\title{
PARTLY INVARIANT SUBMODULES OF A TORSION MODULE
}

BY

\section{R. A. BEAUMONT AND R. S. PIERCE}

1. Introduction. Let $M$ be a torsion module over a principal ideal ring $R$. Kaplansky in [2] has examined the submodules of $M$ which are fully invariant (i.e., carried into themselves by every endomorphism of $M$ ) and those which are characteristic, or invariant under automorphisms of $M$. We will be concerned with a class of submodules which is intermediate between characteristic and fully invariant, namely, those which are partly invariant.

Definition. A submodule of a module $M$ is called partly invariant in $M$ if it is mapped into itself by every isomorphism of $M$ into $M$.

It is clear that any partly invariant submodule is characteristic and any fully invariant submodule is partly invariant. For primary modules, the three concepts usually coincide. Indeed, Kaplansky has shown that if $M$ is primary and transitive (see [2]), and if $R /(p)$ is not a field of two elements, then every characteristic submodule is fully invariant. Moreover, under these hypotheses, the fully invariant submodules are completely determined by their $U$ sequences. But if $R /(p)$ is a field of two elements, Kaplansky's theory leaves room for further study and in this situation the following results are significant.

Our aim is to determine all partly invariant submodules of $M$. The study is quickly reduced to the primary case. Then, subject to the assumption that $M$ is a partly transitive primary module, this objective is achieved as follows: Let $\left(\alpha_{0}, \alpha_{1}, \alpha_{2}, \cdots, \alpha_{N-1}\right)$ be a finite $U$-sequence of ordinals less than $\mu$ and let $T$ be a characteristic submodule of the top submodule of $M$ (the terminology is explained below). Let

$$
S=\left[x \in M \mid h\left(p^{i} x\right) \geqq \alpha_{i} \text { for } 0 \leqq i<N \quad \text { and } \quad p^{N} x \in T\right] .
$$

Then $S$ is a partly invariant submodule of $M$. The principal result of the paper is that, conversely, every partly invariant submodule of $M$, not of this form, must be fully invariant. Taken together with Kaplansky's survey (in [2]) of the fully invariant submodules of $M$, it is reasonable to say that we know all partly invariant submodules of $M$. The main tool in the investigation is the concept of partial transitivity introduced in [1]. The assumption that $M$ is partly transitive looks rather restrictive, but at least is satisfied if $M$ is countably generated (see Theorem 7 in [1]).

2. Prelininaries. Without further ado, let us reduce our problem to the case of prinary modules. This is accomplished by the following result, the elementary proof of which is omitted.

Presented to the Society June 15, 1957; received by the editors July 1, 1957. 
Proposition 1. Let $M$ be a torsion module and $S$ a submodule of $M$. Suppose $M=\sum \oplus M_{p}$ is the primary decomposition of $M$. Let $S_{p}=M_{p} \cap S$. Then $S$ is partly invariant in $M$ if and only if every $S_{p}$ is partly invariant in $M_{p}$.

Henceforth, it will be assumed that $M$ is a $p$-primary module which is partly transitive (see below). It does not seem to be appropriate to split $M$ into its reduced and divisible parts. The trouble is that a direct summand of a partly transitive module may conceivably not be partly transitive. For example, it is easy to show (using Theorem 7 of [1]) that the direct sum of a "locally countable" primary module (see $[2$, p. 58]) and a divisible primary module of infinite rank is partly transitive, but we do not know that every locally countable module is partly transitive. Indeed, the existence in $M$ of a divisible primary submodule of infinite rank means that any two elements $x$ and $y$ of $M$ can be embedded in a countably generated direct summand $N$ whose top submodule is zero. (See the terminology below.) Because of this last property, $U(x) \prec U(y)$ in $M$ implies $U(x) \prec U(y)$ in $N$. Since $N$ is partly transitive by Theorem 7 of [1], there is an isomorphism $\theta$ of $N$ such that $\theta(x)=y$. Obviously $\theta$ can be extended to an isomorphism of $M$.

Before beginning the study of primary modules, it is convenient to collect some basic definitions and terminology. Define $M_{\alpha}$ inductively for ordinals $\alpha$ by $M_{0}=M, M_{\alpha+1}=p M_{\alpha}$ and $M_{\alpha}=\bigcap_{\beta<\alpha} M_{\beta}$ when $\alpha$ is a limit ordinal. Let $\lambda$ be the least ordinal such that $M_{\lambda}=M_{\lambda+1}$. Then $M_{\lambda}$ is the maximal divisible submodule $D$ of $M_{0}$. The ordinal $\lambda$ is called the length of $M$. For $x \in M, x \neq 0$ the height of $x$ (denoted by $h(x)$ ) is the largest ordinal $\alpha \leqq \lambda$ such that $x \in M_{\alpha}$. By convention, $h(0)=\infty$, where $\infty$ exceeds every ordinal. Let $P$ be the set of $x$ of order $p$ in $M$. Define $P_{\alpha}=M_{\alpha} \cap P$. The $\alpha$ th Ulm invariant of $M$ is then $f(\alpha)=\operatorname{dim}\left(P_{\alpha} / P_{\alpha+1}\right)$ for $\alpha<\lambda, f(\lambda)=\operatorname{dim} P_{\lambda}$ and $f(\infty)=0$. Let $\mu$ be the least ordinal such that $\sum_{\mu \leqq \alpha \leqq \lambda} f(\alpha)<\boldsymbol{\aleph}_{0}$ if $f(\lambda)<\boldsymbol{\aleph}_{0}$ and put $\mu=\infty$ otherwise. Define $H=M_{\mu}(=0$ if $\mu=\infty)$. We call $H$ the top submodule of $M$. If $x$ is any element of $M$, define the Ulm sequence of $x$ to be the sequence of ordinals $U(x)=\left(h(x), h(p x), \quad h\left(p^{2} x\right), \cdots\right)$. Then (see [2, Lemma 22]) $U(x)$ $=\left(\alpha_{0}, \alpha_{1}, \alpha_{2}, \cdots, \alpha_{n}, \lambda, \lambda, \cdots, \lambda, \infty, \infty, \cdots\right)$, where $\alpha_{0}<\alpha_{1}<\alpha_{2}<\cdots$ $<\alpha_{n}<\lambda$ and $\alpha_{i}+1<\alpha_{i+1}$ only if $f\left(\alpha_{i}\right) \neq 0$. Moreover, if $f(\lambda)=0$, then there are no $\lambda$ 's in $U(x)$.

Definition. A finite or infinite sequence $U=\left(\alpha_{0}, \alpha_{1}, \alpha_{2}, \cdots, \alpha_{n}, \cdots\right)$ of ordinals (together with the symbol $\infty$ ) is called a $U$-sequence for the module $M$ if

(i) either $\alpha_{i} \leqq \lambda$, or $\alpha_{i}=\infty$,

(ii) $\alpha_{i} \leqq \alpha_{i+1}$ and $\alpha_{i}<\alpha_{i+1}$ if $\alpha_{i}<\lambda$,

(iii) $f\left(\alpha_{i}\right)=0$ implies $\alpha_{i+1}=\alpha_{i}+1$.

A gap is said to occur in $U$ between $\alpha_{i}$ and $\alpha_{i+1}$ if $\alpha_{i}+1<\alpha_{i+1}$. By (iii), this can only happen if $f\left(\alpha_{i}\right) \neq 0$. In particular, if $\lambda$ appears in a $U$-sequence, $f(\lambda) \neq 0$. Also, if $\alpha_{i}$ is the last term of a finite $U$-sequence, (iii) is to be construed as requiring $f\left(\alpha_{i}\right) \neq 0$. Note that the empty sequence is considered to 
be a $U$-sequence, since it satisfies (i), (ii) and (iii) vacuously.

The Ulm sequence of any element of $M$ is a $U$-sequence. The following converse will be needed. It is a slight generalization of Lemma 24 of [2].

Lemma 1. Let $U=\left(\alpha_{0}, \alpha_{1}, \cdots, \alpha_{n-1}, \alpha_{n}, \cdots, \alpha_{m}, \infty, \cdots\right)$ be an infinite $U$-sequence. Suppose $z$ is a given element of $M$ with $U(z)=\left(\alpha_{n}, \cdots, \alpha_{m}\right.$, $\infty, \cdots)$. Then there exists $x \in M$ such that $U(x)=U$ and $p^{n} x=z$.

Proof. Write $M=N \oplus D$, where $N$ is reduced and $D$ is divisible. Correspondingly, $z=y+d$, where $y \in N, d \in D$. If $y \neq 0$, choose $y^{\prime} \in N, d^{\prime} \in D$ such that $p^{n} y^{\prime}=y, p^{n} d^{\prime}=d$ and $U\left(y^{\prime}\right)=U$. The element $d^{\prime}$ exists because $D$ is divisible and the existence of $y^{\prime}$ is a consequence of the proof of Lemma 24 in [2]. Put $x=y^{\prime}+d^{\prime}$. If $y=0$ and $\alpha_{0} \geqq \lambda$, choose $x$ to be any element of $D$ satisfying $p^{n} x=d$. Otherwise, $\alpha_{k}<\lambda, \alpha_{k+1} \geqq \lambda$ for some $k<n$. Then $f\left(\alpha_{k}\right) \neq 0$. For either there is a gap between $\alpha_{k}$ and $\alpha_{k+1}$, or $\alpha_{k}=\lambda-1$. But $f(\lambda-1) \neq 0$, because $\lambda$ is the length of $N$ and $N$ is a reduced module which cannot be 0 , since otherwise $\lambda=0$. Therefore $U^{\prime}=\left(\alpha_{0}, \alpha_{1}, \cdots, \alpha_{k}, \infty, \infty, \cdots\right)$ is a $U$ sequence for $N$. By Lemma 24 of [2], there exists $y^{\prime \prime} \in N$ with $U\left(y^{\prime \prime}\right)=U^{\prime}$ and since $D$ is divisible there exists $d^{\prime \prime} \in D$ such that $p^{n} d^{\prime \prime}=d$. Then $p^{k+1} y^{\prime \prime}=0$, so that $p^{n} y^{\prime \prime}=0$. Hence $x=y^{\prime \prime}+d^{\prime \prime}$ has the required properties.

If $U_{1}=\left(\alpha_{i}\right)$ and $U_{2}=\left(\beta_{i}\right)$, it is customary to write $U_{1} \leqq U_{2}$ if $\alpha_{i} \leqq \beta_{i}$ for all $i$. The module $M$ is called fully transitive if for any $x$ and $y$ in $M$ such that $U(x) \leqq U(y)$, there is an endomorphism $\eta$ of $M$ such that $\eta(x)=y$. The concept of partial transitivity is based on a similar partial ordering of $U$-sequences.

Definition. Let $U_{1}=\left(\alpha_{0}, \alpha_{1}, \alpha_{2}, \cdots\right), U_{2}=\left(\beta_{0}, \beta_{1}, \beta_{2}, \cdots\right)$ be infinite $U$-sequences. Then write

$$
U_{1} \prec U_{2} \quad\left(\text { or } U_{2}>U_{1}\right)
$$

if $\alpha_{i} \leqq \beta_{i}$ for all $i$ and $\alpha_{i}=\beta_{i}$ if $\beta_{i} \geqq \mu$.

If $x \in M$ and $\theta$ is any isomorphism of $M$ into itself, $U(x) \prec U(\theta x)$ (see [1]). This fact motivates the

Definition. A primary module $M$ is called partly transitive if, for any two elements $x$ and $y$ such that $U(x) \prec U(y)$, there is an isomorphism $\theta$ of $M$ into itself sending $x$ into $y$.

3. The basic lemmas. Most of the technical work in the proofs of the main theorems can be collected into two lemmas. In this section, we state and prove these lemmas. For the first result, it is only necessary to assume that $M$ is a primary $R$-module.

Lemma 2. Suppose $x, y \in M$ have Ulm sequences

$$
\begin{aligned}
& U(x)=\left(\alpha_{0}, \alpha_{1}, \cdots, \alpha_{j}, \alpha_{j+1}, \cdots, \alpha_{m}, \infty, \cdots\right), \\
& U(y)=\left(\beta_{0}, \beta_{1}, \cdots, \beta_{j}, \beta_{j+1}, \cdots, \beta_{n}, \infty, \cdots\right),
\end{aligned}
$$

where $f\left(\alpha_{j}\right) \neq 0$. Then there is an element $w \in M$ such that 
(a) $p^{i+1} w=0$,

(b) $U(x) \leqq U(w)$,

(c) $U(y+w)=\left(\gamma_{0}, \gamma_{1}, \cdots, \gamma_{j}, \beta_{j+1}, \cdots, \beta_{n}, \infty, \cdots\right)$, where $\gamma_{i} \leqq \alpha_{i}$ for $0 \leqq i \leqq j$.

Moreover, if also $\alpha_{k} \leqq \beta_{k}, \alpha_{k+1} \leqq \beta_{k+1}, \cdots, \alpha_{j} \leqq \beta_{j}(k \leqq j)$, then w can be chosen so that, in addition to (a), (b) and (c),

(d) $\gamma_{k}=\alpha_{k}, \gamma_{k+1}=\alpha_{k+1}, \cdots, \gamma_{j}=\alpha_{j}$.

Proof. Use induction on $j$. Assume the lemma is true for subscripts $<j$. Let $u$ be the smallest integer $\leqq j$ such that $f\left(\alpha_{i}\right)=0$ for $u \leqq i<j$. (In particular, $u=j$ when $j=0$ or $f\left(\alpha_{j-1}\right) \neq 0$.) There are two possibilities to consider.

CASE I. $\beta_{j} \leqq \alpha_{j}$. Then $\beta_{i} \leqq \alpha_{i}$ for $u \leqq i \leqq j$. If $\alpha_{j}=\lambda$, this is clear since either $j=0, \alpha_{j-1}=\lambda, \alpha_{j-1}=\lambda-1$, or there is a gap between $\alpha_{j-1}$ and $\alpha_{j}$. In any of the last three cases $f\left(\alpha_{j-1}\right) \neq 0$ (see the proof of Lemma 1) and hence $u=j$. If $\alpha_{j}<\lambda$, then the sequence $\left(\alpha_{u}, \alpha_{u+1}, \cdots, \alpha_{j}\right)$ is free from gaps and $\beta_{j} \leqq \alpha_{j}<\lambda$, so that

$$
\beta_{i}+(j-i) \leqq \beta_{j} \leqq \alpha_{j}=\alpha_{i}+(j-i)
$$

for $u \leqq i \leqq j$.

If $u=0$, take $w=0$. Conditions (a), (b), and (c) are clearly satisfied and so is (d) because the hypotheses require $\alpha_{i}=\beta_{i}$ for $k \leqq i \leqq j$ in this case.

If $u>0$, then $f\left(\alpha_{u-1}\right) \neq 0$ by the choice of $u$. Apply the induction hypothesis for the case $u-1$. That is, let $w \in M$ be such that $p^{u} w=0, U(w) \geqq U(x)$ and $U(y+w)=\left(\gamma_{0}, \gamma_{1}, \cdots, \gamma_{u-1}, \beta_{u}, \cdots, \beta_{j}, \beta_{j+1}, \cdots, \beta_{n}, \infty, \cdots\right)$ with $\gamma_{i} \leqq \alpha_{i}$ for $0 \leqq i \leqq u-1$. As noted above, $\beta_{i} \leqq \alpha_{i}$ for $u \leqq i \leqq j$, so that we can define $\gamma_{i}=\beta_{i}$ for $u \leqq i \leqq j$ and satisfy (c). Also, under the hypothesis of (d), if $k \geqq u$, then the conclusion of (d) holds automatically. If $k<u$, it is possible, according to the induction hypothesis, to choose $w$ so that $\gamma_{k}=\alpha_{k}, \cdots, \gamma_{u-1}$ $=\alpha_{u-1}$. But also $\gamma_{i}=\beta_{i}=\alpha_{i}$ for $u \leqq i \leqq j$.

CASE II. $\beta_{j}>\alpha_{j}$. Since $f\left(\alpha_{j}\right) \neq 0, U=\left(\alpha_{0}, \alpha_{1}, \cdots, \alpha_{j}, \infty, \cdots\right)$ is a $U$ sequence. By Lemma 1, there exists $w_{1} \in M$ such that $U\left(w_{1}\right)=U$. Then $p^{j+1} w_{1}=0$ and $h\left(p^{j}\left(y+w_{1}\right)\right)=\alpha_{j}$ since $\alpha_{j}<\beta_{j}$. Thus

$$
U\left(y+w_{1}\right)=\left(\beta_{0}^{\prime}, \beta_{1}^{\prime}, \cdots, \beta_{j-1}^{\prime}, \alpha_{j}, \beta_{j+1}, \cdots, \beta_{n}, \infty, \cdots\right),
$$

where $\beta_{i}^{\prime} \geqq \min \left[\beta_{i}, \alpha_{i}\right]$. Hence, if $\alpha_{i} \leqq \beta_{i}$ for $k \leqq i \leqq j-1$, then also $\alpha_{i} \leqq \beta_{i}^{\prime}$ in this range. Now apply the result of Case I to $y+w_{1}$, getting $w_{2} \in M$ such that $p^{j+1} w_{2}=0, U\left(w_{2}\right) \geqq U(x)$ and $U\left(y+w_{1}+w_{2}\right)=\left(\gamma_{0}, \gamma_{1}, \cdots, \gamma_{j-1}, \alpha_{j}, \beta_{j+1}, \cdots\right.$, $\left.\beta_{n}, \infty, \cdots\right)$, where $\gamma_{i} \leqq \alpha_{i}$ for $i \leqq j-1$ and $\gamma_{i}=\alpha_{i}$ for $k \leqq i \leqq j-1$ (if $\alpha_{i} \leqq \beta_{i}$, and hence $\alpha_{i} \leqq \beta_{i}^{\prime}$, for $\left.k \leqq i \leqq j-1\right)$. Setting $w=w_{1}+w_{2}$ gives the required element, since $U(w) \geqq \min \left[U\left(w_{1}\right), U\left(w_{2}\right)\right] \geqq U(x)$ and $p^{j+1} w=p^{j+1} w_{1}+p^{i+1} w_{2}=0$.

We now assume that $M$, in addition to being primary, is partly transitive.

Lemma 3. Let $x \in M$ have Ulm sequence

$$
U(x)=\left(\alpha_{0}, \alpha_{1}, \cdots, \alpha_{j}, \alpha_{j+1}, \cdots, \alpha_{m}, \infty, \cdots\right),
$$


where $\alpha_{j}<\mu$. Let $y \in M$ satisfy

$$
U(y)=\left(\beta_{0}, \beta_{1}, \cdots, \beta_{j}, \infty, \cdots\right),
$$

where $\beta_{i} \geqq \alpha_{i}$ for $0 \leqq i \leqq j$. Then $y$ is contained in the partly invariant submodule generated by $x$.

Proof. Let $N$ be the partly invariant submodule generated by $x$. We have $U(x+y)=\left(\alpha_{0}^{\prime}, \alpha_{1}^{\prime}, \cdots, \alpha_{j}^{\prime}, \alpha_{j+1}, \cdots, \alpha_{m}, \infty, \cdots\right)$, where $\alpha_{i}^{\prime} \geqq \min \left[\alpha_{i}, \beta_{i}\right]$ $=\alpha_{i}$ for $0 \leqq i \leqq j$.

If $\alpha_{j+1}<\mu$ or if $\alpha_{j}<\beta_{j}$ (so that $\alpha_{j}^{\prime}=\alpha_{j}<\mu$ ), then $U(x)$ and $U(x+y)$ coincide in all terms $\alpha_{i} \geqq \mu$ and $U(x) \prec U(x+y)$. By the partial transitivity of $M$, there exists an isomorphism $\theta$ of $M$ into itself such that $\theta(x)=x+y$. Hence $x+y \in N$ and $y \in N$.

Thus we may assume that $\alpha_{j+1} \geqq \mu$ and that $\alpha_{j}=\beta_{j}$. Consider three cases.

CAsE I. $\mu=\infty$. Then $U(x) \prec U(y)$. By partial transitivity, an isomorphism $\theta$ of $M$ exists such that $\theta(x)=y$. Thus, $y \in N$.

CASE II. $\alpha_{j}+1<\mu<\infty$. Then $\sum_{\alpha_{j}<\gamma<\mu} f(\gamma) \geqq \boldsymbol{\aleph}_{0}$, so that there exists $\gamma_{j}>\alpha_{j}=\beta_{j}$ with $\gamma_{j}<\mu$ and $f\left(\gamma_{j}\right) \neq 0$. Choose a decreasing sequence of ordinals $\gamma_{j-1}, \gamma_{j-2}, \cdots, \gamma_{0}$ such that $\gamma_{i}>\beta_{i}$ for $i<j$ and

$$
U=\left(\gamma_{0}, \gamma_{1}, \cdots, \gamma_{j}, \alpha_{j+1}, \cdots, \alpha_{m}, \infty, \cdots\right)>U(x)
$$

is a $U$-sequence. By Lemma 1 , there exists $w \in M$ such that $U(w)=U$. Also, by the construction of $U$,

$$
U(y+w)=\left(\beta_{0}, \beta_{1}, \cdots, \beta_{j}, \alpha_{j+1}, \cdots, \alpha_{m}, \infty, \cdots\right)>U(x) .
$$

Then by the partial transitivity of $M, w \in N, y+w \in N$ and therefore $y \in N$.

CASE III. $\alpha_{j+1}=\mu<\infty$. By definition of $\mu, f(\mu-1) \geqq \boldsymbol{\aleph}_{0}$. In particular, $f(\mu-1) \geqq 2$, and the definition of the Ulm invariants implies that there exists $w_{1} \in M$ such that $p w_{1}=0$ and $h\left(w_{1}\right)=h\left(p^{i} y+w_{1}\right)=\mu-1=\alpha_{j}$. By Lemma 1, there is an element $w \in M$ such that $U(w)=U(y)$ and $p^{i} w=w_{1}$. Then $h\left(p^{j}(y+w)\right)=\alpha_{j}$.

Consider $h\left(p^{j}(x+y)\right)$ and $h\left(p^{j}(x-w)\right)$. These ordinals are $\geqq \alpha_{j}$ and one of them must be $\alpha_{j}$, since otherwise $h\left(p^{i}(y+w)\right) \geqq \min \left[h\left(p^{j}(x+y)\right), h\left(p^{i}(x-w)\right)\right]$ $>\alpha_{j}$. If $h\left(p^{j}(x+y)\right)=\alpha_{j}$, then

$$
U(x+y)=\left(\alpha_{0}^{\prime}, \alpha_{1}^{\prime}, \cdots, \alpha_{j-1}^{\prime}, \alpha_{j}, \cdots, \alpha_{m}, \infty, \cdots\right)>U(x),
$$

whence $x+y \in N$, so that $y \in N$. Similarly, if $h\left(p^{i}(x-w)\right)=\alpha_{j}$, then $x-w \in N$ and therefore $w \in N$. But $U(w)=U(y)$, so there is an isomorphism $\theta$ of $M$ into itself such that $\theta(w)=y$. Thus $y \in N$ also.

4. The main theorems. Let $\left(\alpha_{0}, \alpha_{1}, \cdots, \alpha_{n-1}\right)$ be a finite $U$-sequence of ordinals less than $\mu$. Let $T$ be a characteristic submodule of $H=M_{\mu}$, the top submodule of $M$.

Define: $S=\left[x \in M \mid h\left(p^{i} x\right) \geqq \alpha_{i}\right.$ for $0 \leqq i<n$ and $\left.p^{n} x \in T\right]$. Extend this definition by letting $S=T$ if $n=0$. 
Proposition 2. $S$ is a partly invariant submodule of $M$. Moreover, $T$ $=p^{n} S \subseteq H, p^{n-1} S \Phi H$ (if $n>0$ ) and

$$
\alpha_{i}=\min \left[h\left(p^{i} x\right) \mid x \in S\right] \quad \text { for } 0 \leqq i<n .
$$

Proof. It is clear that $S$ is a submodule of $M$. If $\theta$ is an isomorphism of $M$ into itself, then $\theta$ restricted to $H$ is an automorphism (see [1, Theorem 6]). Since $T$ is characteristic in $H, \theta T=T$. Thus, for any $x \in S, h\left(p^{i} \theta x\right) \geqq h\left(p^{i} x\right)$ $\geqq \alpha_{i}$ for $0 \leqq i<n$ and $p^{n} \theta x=\theta p^{n} x \in \theta T=T$. Consequently, $\theta x \in S$. This proves that $S$ is partly invariant.

By the definition of $S, p^{n} S \subseteq T \subseteq H$ and $\alpha_{i} \leqq \min \left[h\left(p^{i} x\right) \mid x \in S\right]$ for $0 \leqq i<n$. On the other hand, by Lemma $1, M$ contains an element $x$ whose Ulm sequence is $\left(\alpha_{0}, \alpha_{1}, \cdots, \alpha_{n-1}, \infty, \cdots\right)$. Then by definition, $x \in S$. Hence, $\alpha_{i} \geqq \min \left[h\left(p^{i} x\right) \mid x \in S\right]$ for $0 \leqq i<n$ and $p^{n-1} S \Phi H$ because $h\left(p^{n-1} x\right)$ $=\alpha_{n-1}<\mu$. Finally, suppose $t \in T$. Let $U(t)=\left(\beta_{0}, \beta_{1}, \cdots, \beta_{m}, \infty, \cdots\right)$. Then $U=\left(\alpha_{0}, \alpha_{1}, \cdots, \alpha_{n-1}, \beta_{0}, \cdots, \beta_{m}, \infty, \cdots\right)$ is a $U$-sequence since $\alpha_{n-1}<\mu \leqq \beta_{0}$ and $f\left(\alpha_{n-1}\right) \neq 0$. By Lemma 1 , there exists $y \in M$ such that $p^{n} y=t$ and $U(y)=U$. Then $y \in S$, so $t \in p^{n} S$. Hence, $T=p^{n} S$.

Our first main theorem is a converse of Proposition 2.

Theorem 1. Let $S$ be a partly invariant submodule of the partly transitive, primary module $M$. Let $H$ be the top submodule of $M$. Suppose $p^{n} S \subseteq H$ and $n$ is the least exponent with this property. Define

$$
\begin{aligned}
\alpha_{i} & =\min \left[h\left(p^{i} x\right) \mid x \in S\right], & 0 \leqq i<n, \\
T & =p^{n} S . &
\end{aligned}
$$

Then $\left(\alpha_{0}, \alpha_{1}, \cdots, \alpha_{n-1}\right)$ is a $U$-sequence of ordinals less than $\mu, T$ is a characteristic submodule of $H$ and

$$
S=\left[x \in M \mid h\left(p^{i} x\right) \geqq \alpha_{i} \text { for } 0 \leqq i<n \text { and } p^{n} x \in T\right] .
$$

Proof. We first show that $\left(\alpha_{0}, \alpha_{1}, \cdots, \alpha_{n-1}\right)$ is a $U$-sequence of ordinals less than $\mu$. If $n=0$, this is vacuously true. Otherwise, $p^{n-1} S \Phi H$, so that $h\left(p^{n-1} x\right)<\mu$ for some $x \in S$. Thus, $\alpha_{n-1}<\mu$. It is easy to check that $\alpha_{0}<\alpha_{1}$ $<\cdots<\alpha_{n-1}$ and that $f\left(\alpha_{i}\right)=0$ implies $\alpha_{i+1}=\alpha_{i}+1$ for $i<n-1$ (see [2, Lemma 26]). To see that $f\left(\alpha_{n-1}\right) \neq 0$, suppose $x \in S$ satisfies $h\left(p^{n-1} x\right)=\alpha_{n-1}$. Then $h\left(p^{n} x\right) \geqq \mu$ and if $\alpha_{n-1}+1<\mu$, there is a gap between $h\left(p^{n-1} x\right)$ and $h\left(p^{n} x\right)$ in $U(x)$, so that $f\left(\alpha_{n-1}\right) \neq 0$. Otherwise $\alpha_{n-1}=\mu-1$ and $f\left(\alpha_{n-1}\right)=f(\mu-1) \geqq \boldsymbol{\aleph}_{0}$ by the definition of $\mu$.

We next show that $T$ is a characteristic submodule of $H$. (Note that $T$ is obviously characteristic in $M$.) It is enough to prove the following: if $t \in T$ and $t^{\prime} \in H$ have the same Ulm sequences in $H$, then $t^{\prime} \in T$ also. But for any $z \in H$, if the height of $z$ in $H$ is $k$, then $h(z)=\mu+k$. Indeed, $h(z)=\mu+k$ if and only if $\mu+k<\lambda$ and $z \in M_{\mu+k}-M_{\mu+k+1}=H_{k}-H_{k+1}$, or $\mu+k=\lambda$ and $z \in M_{\lambda}$ $=H_{k}$. Thus, if $t$ and $t^{\prime}$ have the same Ulm sequences in $H$, their Ulm sequences 
in $M$ are equal. By assumption, $t=p^{n} x$ for some $x \in S$. By Lemma 1 , there exists $x^{\prime} \in M$ with

$$
U\left(x^{\prime}\right)=U(x) \text { and } p^{n} x^{\prime}=t^{\prime} .
$$

Since $M$ is partly transitive, there is an isomorphism $\theta$ of $M$ sending $x$ into $x^{\prime}$. Then because $S$ is partly invariant, $x^{\prime}=\theta(x) \in S$. Hence, $t^{\prime}=p^{n} x^{\prime} \in p^{n} S=T$, which is the desired conclusion.

It remains to prove that $S=\bar{S}$, where $\bar{S}$ is defined to be

$$
\left[x \in M \mid h\left(p^{i} x\right) \geqq \alpha_{i} \text { for } 0 \leqq i<n \text { and } p^{n} x \in T\right] .
$$

By the definition of the $\alpha_{i}$ and $T$, it is clear that $S \subseteq \bar{S}$. As the first step in proving that $\bar{S} \subseteq S$, we show that if $t$ is any element of $T$, there exists $x \in S$ such that $p^{n} x=t$ and $h\left(p^{i} x\right)=\alpha_{i}$ for $0 \leqq i<n$.

Let $W$ be the collection of all $x \in S$ such that $p^{n} x=t$. This set is nonempty by the definition of $T$. By the well ordering of the integers, there is a least $m$ $(0 \leqq m \leqq n)$ such that either $m=n$, or $m<n$ and some $x \in W$ satisfies $h\left(p^{i} x\right)$ $=\alpha_{i}$ for $m \leqq i<n$. If $m=0$, the required $x$ is at hand. Suppose $m>0$ and choose $x \in W$ accordingly: $p^{n} x=t$ and

$$
U(x)=\left(\beta_{0}, \beta_{1}, \cdots, \beta_{m-1}, \alpha_{m}, \cdots, \alpha_{n-1}, \delta_{n}, \cdots, \delta_{r}, \infty, \cdots\right) .
$$

We first note that since $\left(\alpha_{0}, \alpha_{1}, \cdots, \alpha_{n-1}\right)$ is a $U$-sequence, $f\left(\alpha_{m-1}\right) \neq 0$. This is clear if $m=n$. Thus, suppose $m<n$. By assumption, $\alpha_{m-1}<\beta_{m-1}$. If $\beta_{m-1}<\lambda$, then $\beta_{m-1}<\alpha_{m}$ and there is a gap between $\alpha_{m-1}$ and $\alpha_{m}$. If $\beta_{m-1}=\lambda$, then either there is a gap between $\alpha_{m-1}$ and $\beta_{m-1}$ (and hence $\alpha_{m}$ ), or $\alpha_{m-1}=\lambda-1$. In each of these cases, $f\left(\alpha_{m-1}\right) \neq 0$, recalling the argument used in Lemma 1 to show $f(\lambda-1) \neq 0$.

By the definition of $\alpha_{m-1}$, it is possible to choose $y \in S$ so that $h\left(p^{m-1} y\right)$ $=\alpha_{m-1}$. Apply Lemma 2(d) (interchanging the roles of $x$ and $y$ and noting $\left.\alpha_{m-1}<\beta_{m-1}\right)$ to get $w \in M$ such that

$$
\begin{aligned}
p^{m} w & =0, \quad U(w) \geqq U(y), \\
U(x+w) & =\left(\gamma_{0}, \gamma_{1}, \cdots, \gamma_{m-2}, \gamma_{m-1}, \alpha_{m}, \cdots, \alpha_{n-1}, \delta_{n}, \cdots \delta_{r}, \infty, \cdots\right),
\end{aligned}
$$

where $\gamma_{m-1}=\alpha_{m-1}$. By (1) and Lemma $3, w \in S$. Thus $x+w \in S$ and $p^{n}(x+w)$ $=p^{n} x=t$, so $x+w \in W$. But then (2) contradicts the minimal property of $m$. Hence, we are forced to the desired conclusion $m=0$.

Now let $y \in \bar{S}$. Then $t=p^{n} y \in T$ and if

$$
U(y)=\left(\beta_{0}, \beta_{1}, \cdots, \beta_{n-1}, \delta_{n}, \cdots, \delta_{r}, \infty, \cdots\right),
$$

$\beta_{i} \geqq \alpha_{i}$ for $0 \leqq i<n$. As we have just seen, there exists $x \in S$ with $p^{n} x=t=p^{n} y$ and with $h\left(p^{i} x\right)=\alpha_{i}$ for $0 \leqq i<n$. Thus

$$
C(r)=\left(\alpha_{0}, \alpha_{1}, \cdots, \alpha_{n-1}, \delta_{n}, \cdots, \delta_{r}, \infty, \cdots\right) .
$$


Since $f\left(\alpha_{n-1}\right) \neq 0$, Lemma 2 can be applied to get $w \in M$ such that

$$
\begin{aligned}
p^{n} w & =0, \quad U(w) \geqq U(x) \text { and } \\
U(y+w) & =U(x) .
\end{aligned}
$$

By (3) and Lemma 3, $w \in S$ and by (4) and the partial transitivity, $y+w \in S$. Hence, $y \in S$. Thus $\bar{S} \subseteq S$ and the proof of Theorem 1 is complete.

Proposition 2 and Theorem 1 characterize those partly invariant submodules $S$ with the property that $p^{n} S \subseteq H$ for some exponent $n$. Our second main theorem shows that, aside from the fully invariant ones, these are all of the partly invariant submodules of $M$.

THEOREM 2. Let $S$ be a partly invariant submodule of the partly transitive primary module $M$. Denote by $H$ the top submodule of $M$. Suppose $p^{n} S \Phi H$ for all exponents $n$. Then $S$ is fully invariant.

Proof. If $\eta$ is any endomorphism of $M$ and $x \in M$, then $U(\eta x) \geqq U(x)$. Thus, it suffices to show that if $x \in S, y \in M$ and $U(y) \geqq U(x)$, then $y \in S$. Let

$$
\begin{aligned}
& U(x)=\left(\alpha_{0}, \alpha_{1}, \cdots, \alpha_{j}, \alpha_{j+1}, \cdots, \alpha_{m}, \infty, \cdots\right) \text { and } \\
& U(y)=\left(\beta_{0}, \beta_{1}, \cdots, \beta_{j}, \beta_{j+1}, \cdots, \beta_{n}, \infty, \cdots\right),
\end{aligned}
$$

where $\alpha_{j}<\mu \leqq \alpha_{j+1}$ and $\alpha_{i} \leqq \beta_{i}$ for $0 \leqq i \leqq n \leqq m$. If $n \leqq j$, then $y \in S$ by Lemma 3 . Therefore, assume $n>j$. By assumption, $p^{n} S \Phi H$, so there exists $z \in S$ with $p^{n} z \notin H$ and hence,

$$
U(z)=\left(\gamma_{0}, \gamma_{1}, \cdots, \gamma_{j}, \gamma_{j+1}, \cdots, \gamma_{n}, \gamma_{n+1}, \cdots\right),
$$

with $\gamma_{n}<\mu$. Noting $f\left(\alpha_{j}\right) \neq 0$, we can apply Lemma 2 (with $z$ in the role of $y$ ). This yields $w \in M$ such that $p^{i+1} w=0, U(w) \geqq U(x)$ and

$$
U(w+z)=\left(\delta_{0}, \delta_{1}, \cdots, \delta_{j}, \gamma_{j+1}, \cdots, \gamma_{n}, \gamma_{n+1}, \cdots\right),
$$

where $\delta_{i} \leqq \alpha_{i}$ for $0 \leqq i \leqq j$. By Lemma $3, w \in S$ and hence $w+z \in S$. But also, $U(w+z) \leqq U(y)$. Indeed, $\delta_{i} \leqq \alpha_{i}$ for $0 \leqq i \leqq j, \gamma_{i}<\mu \leqq \alpha_{i} \leqq \beta_{i}$ for $j+1 \leqq i \leqq n$, and obviously $\gamma_{i} \leqq \infty=\beta_{i}$ for $i \geqq n+1$. Therefore, Lemma 3 again applies, this time with $w+z$ in the role of $x$. The final conclusion is that $y \in S$ and the proof is complete.

There is one gap in our survey of the partly invariant submodules of $M$, namely, which of the partly invariant submodules constructed in Proposition 2 are actually fully invariant? The following result answers this question in a fairly satisfactory way.

Theorem 3. Let $S$ be a partly invariant submodule of $M$ such that $p^{n} S \subseteq H$, $p^{n-1} S \Phi H$ for some exponent $n$, where $H$ is the top submodule of $M$.

(a) Let $M$ be partly transitive. If $p^{n} S$ is fully invariant in $H$, then $S$ is fully invariant in $M$.

(b) Let $M$ be fully transitive. If $S$ is fully invariant in $M$, then $p^{n} S$ is fully invariant in $H$. 
Proof. By Theorem 1, $S=\left[x \in M \mid h\left(p^{i} x\right) \geqq \alpha_{i}, 0 \leqq i<n\right.$, and $\left.p^{n} x \in T\right]$, with $\left(\alpha_{0}, \alpha_{1}, \cdots, \alpha_{n-1}\right)$ a $U$-sequence of ordinals $<\mu$, and $T=p^{n} S$ is fully invariant in $H$ by hypothesis. Suppose $x \in S, y \in M$ and $U(x) \leqq U(y)$. Then $h\left(p^{i} y\right) \geqq \alpha_{i}$ for $0 \leqq i<n$ and $U\left(p^{n} x\right) \leqq U\left(p^{n} y\right)$ in $H$. By Theorem 6 of [1], $H$ has finite rank. Thus $H$ is countably generated, and it is therefore fully transitive (see [2, Theorem 24]). From the fact that $T$ is fully invariant and $p^{n} x \in T$, it follows that $p^{n} y \in T$. Consequently, $y \in S$ and $S$ is fully invariant.

To prove the converse, note that if $S$ is fully invariant in $M$, then so is $T=p^{n} S$. Hence, we must prove that if $M$ is fully transitive and $T$ is a fully invariant submodule of $M$ which is contained in $H$, then $T$ is fully invariant in $H$. Suppose $t \in T, z \in H$ and there is an endomorphism of $H$ sending $t$ into $z$. Then the Ulm sequence of $t$ in $H$ is less than or equal to the Ulm sequence of $z$ in $H$, which implies $U(t) \leqq U(z)$ in $M$. By full transitivity, there is an endomorphism $\eta$ of $M$ such that $\eta(t)=z$. Since $T$ is fully invariant in $M$, $\eta T \subseteq T$ and hence $z \in T$. Consequently, $T$ is fully invariant in $H$.

COROLlaRy. If a partly transitive module $M$ contains a partly invariant submodule which is not fully invariant, then

(a) $R /(p)$ is a two element field, and

(b) there exist ordinals $\alpha$ and $\beta$ with $\mu \leqq \alpha<\alpha+1<\beta$ such that $f(\alpha)=f(\beta)=1$.

Conversely, if $M$ is fully transitive, and if conditions (a) and (b) are satisfied, then $M$ contains a partly transitive module which is not fully invariant.

Proof. Observe that the Ulm invariants of $H$ are precisely $f(\mu)$, $f(\mu+1), \cdots$. If either of conditions (a) or (b) fails, then by Theorem 26 in [2] every characteristic submodule of $H$ is fully invariant. Now if $S$ is a partly invariant submodule of a partly transitive module $M$, either $p^{n} S \Phi H$ for all exponents $n$, in which case $S$ is fully invariant by Theorem 2 ; or $p^{n} S \subseteq H$ for some $n$. In the latter case, $p^{n} S$ is a characteristic submodule of $H$ by Theorem 1 , and hence by the above remark, $p^{n} S$ is fully invariant in $H$. By (a), Theorem $3, S$ is fully invariant in $M$.

Conversely, if (a) and (b) are satisfied for a fully transitive module $M$, then by Theorem 27 in [2], $H$ contains a characteristic submodule $T$ which is not fully invariant. By Theorem 6(c) of [1], any characteristic submodule of $H$ is partly invariant in $H$. If $H=M$, then every characteristic submodule of $M$ is partly invariant which gives the desired result. If $H \neq M$, then $\mu \neq 0$, and a partly invariant submodule $S$ constructed using Proposition 2 and the given $T$, is not fully invariant by (b) of Theorem 3 .

The above corollary suggests a similar question: when will a module contain characteristic submodules which are not partly invariant? Our last theorem, which is analogous to Theorem 27 of [2], gives a sufficient condition for the existence of such characteristic submodules.

THEOREM 4. Let $M$ be a partly transitive, $p$-primary module, where $R /(p)$ is a two element field. Suppose there exist ordinals $\alpha$ and $\beta$ such that 


$$
\alpha<\mu, \quad \alpha+1<\beta \quad \text { and } f(\alpha)=f(\beta)=1 .
$$

Then $M$ has a characteristic submodule which is not partly invariant.

Proof. Let $S$ be the submodule generated by all $x \in M$ such that $U(x)$ $=(\alpha, \beta, \infty, \cdots)$. Clearly $S$ is a characteristic submodule of $M$. To show that $S$ is not partly invariant, we first prove that $y \in S$ implies $U(y)$ $=(\alpha, \beta, \infty, \cdots)$, or $U(y)=(\gamma, \delta, \infty, \cdots)$, where $\gamma>\alpha$ and $\delta>\beta$. To see this, consider $x_{1}$ and $x_{2}$ such that $U\left(x_{1}\right)=U\left(x_{2}\right)=(\alpha, \beta, \infty, \cdots)$. Then $p x_{1}$ and $p x_{2}$ are of order $p$ and height $\beta$. Thus, since $f(\beta)=1, p x_{1} \equiv p x_{2} \bmod M_{\beta+1}$, and since $R /(p)$ is of characteristic two, $p x_{1}+p x_{2} \in M_{\beta+1}$. That is, $h\left(p\left(x_{1}+x_{2}\right)\right)$ $>\beta$. Also, $h\left(p x_{i}\right)>\alpha+1$ implies $p x_{i}=p y_{i}$, where $h\left(y_{i}\right)>\alpha$. That is, $x_{i}=y_{i}+z_{i}$, where $h\left(y_{i}\right)>\alpha, h\left(z_{i}\right)=\alpha$ and $p z_{i}=0$. As above, since $f(\alpha)=1, h\left(z_{1}+z_{2}\right)>\alpha$, and therefore $h\left(x_{1}+x_{2}\right)>\alpha$. Now the general term of $S$ is $y=\sum c_{k} x_{k}$, where $c_{k} \equiv 0$ or $1 \bmod p$ and $U\left(x_{k}\right)=(\alpha, \beta, \infty, \cdots)$. The above remarks show that $U(y)=(\alpha, \beta, \infty, \cdots)$ if the number of $c_{k} \equiv 1 \bmod p$ is odd and $U(y)$ $=(\gamma, \delta, \infty, \cdots)$ (with $\gamma>\alpha, \delta>\beta)$ if this number is even.

Now consider two possibilities.

CASE I. $\beta<\mu$. Then since $f(\beta)=1$, there exists an ordinal $\zeta$ with $\beta<\zeta<\mu$ such that $f(\zeta) \neq 0$. Take $z \in M$ such that $U(z)=(\alpha, \zeta, \infty, \cdots)$.

CASE II. $\beta \geqq \mu$. Since $f(\alpha)=1$, there exists $\zeta$ with $\alpha<\zeta<\mu$ such that $f(\zeta) \neq 0$. Take $z \in M$ such that $U(z)=(\zeta, \beta, \infty, \cdots)$. In both cases, $z \notin S$, but since $M$ is partly transitive there is an isomorphism of $M$ sending a generator of $S$ into $z$. Hence, $S$ is not partly invariant.

By Theorem 4 and the corollary of Theorem 3, a 2-primary abelian group $G$ which is both partly transitive and fully transitive will contain characteristic subgroups which are not partly invariant and partly invariant subgroups which are not fully invariant, provided that among the Ulm invariants of $G$ there are ordinals $\alpha, \beta$, and $\gamma$, with $\alpha<\mu, \alpha+1<\beta, \mu \leqq \beta, \beta+1<\gamma$, for which $f(\alpha)=f(\beta)=f(\gamma)=1$. Such groups are easy to obtain, since any countable abelian group is partly transitive (see [1]) and fully transitive (see [2]). Moreover, one has considerable freedom in prescribing the Ulm invariants of a countable group. For example, we can require that $f(0)=1, f(n)=2$ for $n>0, f(\omega)=1, f(\omega+1)=0, f(\omega+2)=1, f(\omega+3)=0, \lambda=\omega+3$. Then $\mu=\omega$ and the countable group $G$ with these Ulm invariants satisfies the required conditions (with $\alpha=0, \beta=\omega, \gamma=\omega+2$ ). As a matter of fact, $G$ can be realized as $G_{1} \oplus G_{2}$, where

$$
G_{1}=\left\{v, u_{1}, \cdots, u_{n}, \cdots\right\},
$$

with relations $p^{3} v=0, v=p^{2} u_{1}=\cdots=p^{n+1} u_{n}=\cdots$.

$$
G_{2}=\left\{x, y_{1}, \cdots, y_{n}, \cdots\right\},
$$

with relations $p x=0, x=p y_{1}=\cdots=p^{n} y_{n}=\cdots$. 
For the group $G_{1}$, we have that for $n>0, p^{n} G_{1}=\left\{v, p^{n} u_{n}, p^{n} u_{n+1}, \cdots\right\}$, and it can be checked that for every element

$$
y \in p^{n} G_{1} \cap P_{1}, y \equiv k z \bmod p^{n+1} G_{1} \cap P_{1},
$$

where $k=0,1,2, \cdots, p-1$, and

$$
z=p^{n} u_{n}+p^{n+1} u_{n+1}+\cdots+p^{n+p^{3}-1} u_{n+p^{3}-1} .
$$

The element $z \in p^{n} G_{1} \cap P_{1}$ and $z \notin p^{n+1} G_{1} \cap P_{1}$. Hence for $n>0$,

$$
p^{n} G_{1} \cap P_{1} / p^{n+1} G_{1} \cap P_{1}=\left\{z+p^{n+1} G_{1} \cap P_{1}\right\}
$$

is a cyclic group of order $p$, so that $f(n)=1$. Since $P_{1} \subseteq p G_{1}, G_{1} \cap P_{1} / p G_{1} \cap P_{1}$ $=P_{1} / P_{1}=0$, so that $f(0)=0$. Further $p^{\omega} G=\{v\}, p^{\omega+1} G=\{p v\}, p^{\omega+2} G=\left\{p^{2} v\right\}$, $p^{\omega+3} G=0$ so that clearly $f(\omega)=0, f(\omega+1)=0, f(\omega+2)=1$, and $\lambda=\omega+3$.

For the group $G_{2}$, we have that for $n \geqq 0, p^{n} G_{2}=\left\{x, p^{n} y_{n+1}, p^{n} y_{n+2}, \cdots\right\}$, and it can be verified that for $y \in p^{n} G_{2} \cap P_{2}, y \equiv k w \bmod p^{n+1} G_{2} \cap P_{2}$, where $k=0,1, \cdots, p-1$, and $w=p^{n} y_{n+1}+p^{n+1} y_{n+2}+\cdots+p^{n+n-1} y_{n+p}$. The element $w \in p^{n} G_{2} \cap P_{2}$ and $w \notin p^{n+1} G_{2} \cap P_{2}$. Hence for $n \geqq 0, f(n)=1$. Further $p^{\omega} G_{2}=\{x\}, p^{\omega+1} G_{2}=0$, so that $f(\omega)=1$ and $\lambda=\omega+1$.

As an Ulm invariant of $G=G_{1} \oplus G_{2}$ is the sum of the corresponding Ulm invariants of $G_{1}$ and $G_{2}, G$ has the Ulm invariants which were given above.

If we now consider the case $p=2$, the subgroup $S$ of $G$ generated by the elements $y \in G$ such that $U(y)=(\omega, \omega+2, \infty, \infty, \cdots)$ is partly invariant and not fully invariant. The subgroup $S=\{2 v+x\}$, a cyclic group of order 4 . The subgroup $T$ of $G$ generated by the elements $z \in G$ such that $U(z)$ $=(0, \omega, \infty, \infty, \cdots)$ is characteristic and not partly invariant. The subgroup $T=\left\{y_{1}\right\}$, a cyclic group of order 4 .

\section{REFERENCES}

1. R. A. Beaumont and R. S. Pierce, Partly transitive modules and modules with proper isomorpkic submodules, Trans. Amer. Math. Soc. vol. 91 (1959) pp. 209-219.

2. I. Kaplansky, Infinite abelian groups, University of Michigan Publications in Mathematics, no. 2, 1954.

UNIVERSITY OF WASHINGTON,

Seattle, Wash. 